\title{
Adequação de ciclo e estatura de planta é essencial para a comparação de genótipos de milho ${ }^{1}$
}

\author{
Cycle and plant height arrangement are essential to compare corn genotypes
}

\author{
Lindolfo Storck ${ }^{2}$ Alberto Cargnelutti Filho ${ }^{3}$ Alessandro Dal' Col Lúcio ${ }^{4}$ \\ Paula Machado dos Santos ${ }^{5}$ Melissa Pisaroglo de Carvalho ${ }^{5}$
}

\section{RESUMO}

A falta da comparabilidade dos efeitos nos ensaios de competição de genótipos de milho (Zea mays) pode resultar em recomendações equivocadas. O objetivo deste trabalho foi verificar se o enquadramento dos genótipos de milho quanto ao ciclo é correto e se este altera a comparação das médias. Foram conduzidos seis experimentos de competição de genótipos de milho de ciclo superprecoce, precoce e normal, em dois níveis tecnológicos, na área experimental da UFSM, durante o ano agrícola de 2000/2001. O experimento bifatorial (genótipos nas parcelas principais e densidades nas subparcelas) foi conduzido no delineamento blocos ao acaso com três repetições. A proporção de experimentos de pressuposições adequadas foi alta, indicando análise de boa qualidade. Os genótipos de milho encontram-se mal agrupados quanto ao ciclo e, por conseqüencia, quanto à estatura de plantas, aumentando a heterogeneidade nos experimentos e prejudicando a comparação das médias. Genótipos de milho com ciclo e/ou estatura de plantas diferentes não devem ser comparados na mesma densidade e experimento.

Palavras-chave: população de plantas, rede de ensaios, Zea mays.

\section{ABSTRACT}

Genotype effects can result in recommendation mistakes in corn (Zea mays) competition trials. The objective was to verify if corn genotypes were well arranged and their consequences to mean comparisons. Six experiments were carried out at the experimental area of the Federal University of Santa Maria with early, medium and late cycle corn genotypes during 2000/2001 grown season. The experimental design was a factorial (genotype in main plots and plant density in sub-plots) in a randomized complete block with three replications. The mathematical model assumptions were adequate in most cases. Genotype arrangements were not adequate for cycle and plant height, increasing experimental errors and reducing statistical power of mean comparisons. Corn genotypes with different cycle and/or plant height should not be compared in the same plant density and trial.

Key words: plant population, competition trials, Zea mays.

\section{INTRODUÇÃO}

Os genótipos de milho, antes de serem indicados aos produtores, são avaliados em rede de ensaios de competição de genótipos, sendo classificados, quanto à duração de seu ciclo, em três categorias principais: superprecoce, precoce e normal, de acordo com a temperatura expressa em número de graus dia que cada genótipo requer para florescer (EMBRAPA, 1997; PEREIRA et al., 2001), quando as

\footnotetext{
${ }^{1}$ Auxílio financeiro do Conselho Nacional de Desenvolvimento Científico e Tecnológico (CNPq) e da Fundação de Amparo à Pesquisa Do Estado do Rio Grande do Sul (FAPERGS).

${ }^{2}$ Engenheiro Agrônomo, Doutor, Professor Titular, Departamento de Fitotecnia, Universidade Federal de Santa Maria (UFSM), 97105-900, Santa Maria, RS. E-mail: storck@ccr.ufsm.br (Autor para correspondência). Bolsista do CNPq

${ }^{3}$ Engenheiro Agrônomo, Doutor, Pesquisador em Estatística/Experimentação da Fundação Estadual de Pesquisa Agropecuária (FEPAGRO), Rua Gonçalves Dias, 570, Bairro Menino Deus, 90130-060, Porto Alegre, RS. E-mail: albertocargnelutti@fepagro.rs.gov.br

${ }^{4}$ Engenheiro Agrônomo, Doutor, Professor Adjunto, Departamento de Fitotecnia, UFSM. E-mail: adlucio@ccr.ufsm.br

${ }^{5}$ Acadêmico de Agronomia, UFSM.
} 
superprecoce são caracterizadas por apresentarem exigências térmicas menores que 830 graus dia da emergência até atingir 50\% do florescimento masculino; as precoces, por apresentarem exigências calóricas entre 830 e 880 graus dias; e as normais, exigências térmicas acima de 880 graus dia. A metodologia desses ensaios recomenda, e nem sempre é observado, que os genótipos de milho de cada ciclo devem formar um experimento. Sob o ponto de vista da técnica experimental, existe um confundimento de efeitos, ou melhor, o efeito do genótipo é confundido com os efeitos do nível tecnológico utilizado, como densidade, adubação, irrigação e outros, que deve ser considerada para uma adequada comparação dos genótipos.

Não se pode comparar um genótipo de ciclo superprecoce de porte baixo e folhas mais eretas com outro genótipo de ciclo precoce de porte mais alto usando a mesma densidade de plantas, adubação, disponibilidade de água entre outros, porque um e/ou o outro genótipo estará sendo prejudicado por não estar sendo dadas as condições ideais, às quais este foi melhorado, para expressar todo seu potencial produtivo.

O maior potencial de resposta para rendimento de grãos, em relação ao aumento de densidades, principalmente para os genótipos superprecoces e precoces, tem sido estudado por diversos pesquisadores no sul do Brasil, como mostram os trabalhos de ALMEIDA \& SANGOI (1996), MEROTTO JUNIOR et al. (1997), PEIXOTO et al.(1997), FLESCH \& VIEIRA (1999), MEROTTO JUNIOR et al. (1999), SILVA et al. (1999), ALMEIDA et al. (2000) e SANGOI et al. (2001). Pode-se dizer que os resultados apontam para um maior rendimento em densidades superiores às recomendadas atualmente pela pesquisa no que se refere aos genótipos estudados nos trabalhos. Ainda, observa-se que a densidade ideal dos genótipos varia entre e dentro de ciclos, com tendência de menores densidades para genótipos tardios.

Assim, o fato de se utilizarem densidades iguais para todos os genótipos dentro do mesmo ciclo pode não ser correto, pois existe a possibilidade de alguns genótipos estarem sendo prejudicados por serem avaliados com densidades inadequadas e/ou por erros no enquadramento de ciclo. Como conseqüência, pode-se estar deixando de indicar aos produtores um determinado genótipo, que teria um potencial maior do que aqueles indicados, tornandose necessário comparar os genótipos na condição da densidade ótima de cada um.

O objetivo deste trabalho foi verificar se o enquadramento dos genótipos de milho quanto ao ciclo é correto e se este altera a comparação das médias dos tratamentos.

\section{MATERIAL E MÉTODOS}

Seis experimentos de milho foram conduzidos durante o ano agrícola de 2000/2001, em área do Departamento de Fitotecnia da Universidade Federal de Santa Maria, situada no município de Santa Maria, cujo solo classifica-se como Brunizem Hidromórfico. A semeadura foi realizada manualmente em 31/10/2000, sob o sistema de plantio direto na aveia preta dessecada, colocando-se duas sementes/cova, que foram desbastadas para uma planta em 15/11/2000. Os sulcos de semeadura foram demarcados em 30/10/2000, por semeadora direta que serviu também para a aplicação do adubo de base (490 $\mathrm{kg} \mathrm{ha}^{-1}$ ), tendo a seguinte composição: $6 \%$ de $\mathrm{N}, 19 \%$ de $\mathrm{P}_{2} \mathrm{O}_{5}, 15 \%$ de $\mathrm{K}_{2} \mathrm{O}, 9 \%$ de Ca e $3 \%$ de S.

Três experimentos (ciclo superprecoce, ciclo precoce ciclo normal) foram conduzidos na condição de tecnologia melhorada e três, na condição de tecnologia recomendada pela coordenação dos ensaios (Técnicos da Fundação Estadual de Pesquisa Agropecuária). Na condição de tecnologia melhorada, fizeram-se quatro irrigações suplementares por aspersão (nos períodos com déficit hídrico) e três adubações nitrogenadas de cobertura. Os experimentos na condição de tecnologia recomendada não receberam as irrigações suplementares, com duas adubações de cobertura. Os seis experimentos bifatoriais (genótipos nas parcelas principais e densidades nas subparcelas) foram conduzidos no delineamento em blocos ao acaso, com três repetições. As parcelas principais, formadas por duas filas com 5,0m de comprimento e espaçadas a $0,8 \mathrm{~m}$, foram assim constituídas: Experimento 1: 16 genótipos superprecoce em tecnologia melhorada; Experimento 2: 30 genótipos precoce em tecnologia melhorada; Experimento 3: 9 genótipos normal em tecnologia melhorada; Experimento 4: 16 genótipos superprecoce em tecnologia recomendada; Experimento 5: 30 genótipos precoce em tecnologia recomendada; Experimento 6: 9 genótipos normal em tecnologia recomendada. Nas subparcelas, foram casualizadas duas situações quanto a densidade de plantas: D (plantas ha $^{-1}$ recomendadas em função do ciclo, independentemente do nível tecnológico - 50, 55 e 65 mil plantas ha-1 ${ }^{-1}$ respectivamente para genótipos dos ciclos normal, precoce e superprecoce) e D (plantas $\mathrm{ha}^{-1}$ recomendadas pelos pesquisadores das èmpresas produtoras de sementes) apresentadas na tabela 1.

As variáveis avaliadas foram: rendimento de grãos de milho, ajustado para 13\% de umidade; 
número de dias do subperíodo semeadura a $50 \%$ do pendoamento; estatura de planta e de inserção da primeira espiga (média de cinco plantas); número de plantas e de espigas com grãos na colheita.

Verificaram-se as pressuposições do modelo matemático (normalidade e aleatoriedade do erro, homogeneidade das variâncias e aditividade do modelo) conforme as aplicações de MARQUES (1999) e os resultados das variáveis observadas foram submetidos à análise da variância, aplicando teste F, estimativas das diferenças mínimas significativas pelo teste de Tukey para os efeitos principais e comparação das médias dos genótipos pelo teste de Scott-Knott (RAMALHO et al., 2000), com o uso do programa estatístico SAEG (RIBEIRO JÚNIOR, 2001).

\section{RESULTADOS E DISCUSSÃO}

Nas 36 verificações de pressuposições (seis variáveis em seis experimentos), observou-se que, na totalidade dos casos, os modelos são aditivos e com distribuição normal dos erros, 92\% dos casos têm variância dos erros homogêneos e 89\% possuem erros aleatórios. Portanto, mostrou-se alta a freqüência de experimentos com as pressuposições adequadas e semelhantes aos encontrados por MARQUES (1999), o que permite inferir que a análise do conjunto de experimentos é de boa qualidade.

Das 110 recomendações de densidade $\mathrm{D}_{\mathrm{i}}$ (55 genótipos em tecnologia melhorada e 55 em tecnologia recomendada), referentes aos genótipos avaliados nos seis experimentos de milho, 78 recomendações $D_{i}(71 \%)$ diferiram da densidade $D_{0}$, sendo 35 recomendações com $\mathrm{D}_{\mathrm{i}}>\mathrm{D}_{0}$ e 43 com $\mathrm{D}_{\mathrm{i}}<\mathrm{D}_{0}$ (Tabela 1). Por outro lado, em 32 casos (29\%), a $\mathrm{D}_{\mathrm{i}}$ foi a mesma que a $D_{0}$. As densidades $D_{i}$ variaram de 50 a 70 mil plantas ha ${ }^{-1}$ nos experimentos com tecnologia melhorada e de 45 a 60 mil plantas ha ${ }^{-1}$ naqueles com tecnologia recomendada, enquanto a metodologia recomenda de 50 (normal) a 65 (superprecoce) mil plantas $\mathrm{ha}^{-1}$, independentemente da tecnologia utilizada (Tabela 1). Estas recomendações são semelhantes às citadas por EMBRAPA(1997), porém menores que as indicadas por MEROTTO JUNIOR et al. (1997).

Havendo diferenças entre os genótipos, quanto ao dias para pendoamento, dentro dos ciclos superprecoce, precoce e normal nos dois níveis tecnológicos, acrescidas por diferenças significativas das estaturas de plantas e de espigas (Tabela 2), verifica-se que o agrupamento dos genótipos, quanto ao ciclo, não atende à recomendação da técnica experimental de agrupar tratamentos de comportamentos semelhantes com o objetivo de reduzir a competição interparcelar (STORCK et al., 2000) e, com isto, melhorar a qualidade dos experimentos. Tal agrupamento não pode ser justificado, principalmente, nos ensaios superprecoce e precoce, nos quais as médias de dias para pendoamento são bastante semelhantes e de grande amplitude de variação. Há a possibilidade de existir diferença significativa entre genótipos e de eles serem aceitos como pertencentes ao mesmo ciclo devido ao fato de a diferença mínima significativa $(\Delta g)$ ser baixa (3,48 a 5,28 dias) nos seis experimentos. No entanto, não se justifica a forma de enquadramento desses genótipos, sendo que a amplitude de variação quanto aos dias para pendoamento, dentro dos ciclos, varia de 5,6 a 13,3 dias entre os genótipos dentro do mesmo ciclo. Devese, portanto, reformular o agrupamento de genótipos baseados em faixas, que sejam mutuamente exclusivos, de dias para pendoamento estimados a partir da soma térmica, em graus dias, necessários para o pendoamento, e/ou então agrupar, em função da estatura ou de forma equivalente, por base genética (híbridos simples, triplos e duplos, que guardam semelhanças morfológicas dentro dos mesmos).

Também, a significância da interação genótipo densidade em todos os seis experimentos, para número de plantas (Tabela 2), era esperada e desejada, visto que comprova a eficiência na implantação dos tratamentos (densidades), conforme o estabelecido (Tabela 1) na metodologia. No entanto, esta interação não repercutiu da mesma forma na variável número de espigas e, conseqüentemente, no rendimento de grãos, possivelmente devido aos efeitos de competição e/ou a maior sensibilidade do rendimento à variação ambiental (erro experimental), não atingindo a significância da interação. Por outro lado, existem diferenças de rendimento entre os genótipos nos seis experimentos (Tabelas 2 e 3), com boa precisão, e entre densidades para dois experimentos (Tabelas 2 e 4). É provável que o agrupamento inadequado dos genótipos quanto ao ciclo e/ou estatura de plantas, com efeito sobre a competição interparcelar, tenha mascarado o efeito da interação na variável rendimento de grãos.

A correlação linear entre as densidades $\Delta_{\mathrm{i}}$ e estatura de planta, em cada experimento, foi negativa e significativa em nível de 5\% de probabilidade de erro. Isso indica que as recomendações das empresas foram mais altas para os genótipos com plantas mais baixas. Esses resultados concordam com ALMEIDA \& SANGOI (1996), EMBRAPA (1997), MEROTTO JUNIOR et al. (1997), MEROTTO JUNIOR et al.(1999), ALMEIDA et al. (2000) e SANGOI et al. (2001), os 
Tabela 1 - Nome, número, tipo e empresa produtora de semente dos genótipos utilizados nos experimentos de ciclo superprecoce, precoce e normal, densidade $\mathrm{D}_{0}$ (plantas $\mathrm{ha}^{-1}$ recomendada pela metodologia dos ensaios) e densidade $\mathrm{D}_{\mathrm{i}}$ (plantas ha ${ }^{-1}$ recomendada pelos pesquisadores das empresas produtoras de sementes) para tecnologia recomendada e melhorada. Santa Maria - RS, 2000/2001

\begin{tabular}{|c|c|c|c|c|c|}
\hline Nome & $\mathrm{N}^{\circ}$ & Tipo* & Empresa & $\mathrm{D}_{\mathrm{i}}$ recomendada & $\mathrm{D}_{\mathrm{i}}$ melhorada \\
\hline \multicolumn{6}{|c|}{ Superprecoce $-\mathrm{D}_{0}=65000$ plantas ha ${ }^{-1}$} \\
\hline AS3601 & 1 & HT & AGROESTE & 55000 & 65000 \\
\hline AGN2012 & 2 & HD & AGROMEN & 45000 & 60000 \\
\hline AGN3050 & 3 & HS & AGROMEN & 55000 & 60000 \\
\hline AGN3150 & 4 & HTM & AGROMEN & 55000 & 60000 \\
\hline AGN3511 & 5 & HTM & AGROMEN & 55000 & 60000 \\
\hline CD3211 & 6 & HD & COODETEC & 50000 & 50000 \\
\hline AG6016 & 7 & HT & MONSANTO & 50000 & 60000 \\
\hline AG6018 & 8 & HT & MONSANTO & 55000 & 65000 \\
\hline C806 & 9 & $\mathrm{HT}$ & MONSANTO & 50000 & 60000 \\
\hline C909 & 10 & HS & MONSANTO & 50000 & 60000 \\
\hline C929 & 11 & HSM & MONSANTO & 60000 & 70000 \\
\hline FLASH & 12 & HS & NOVARTIS & 60000 & 70000 \\
\hline 32R21 & 13 & HS & PIONEER & 50000 & 55000 \\
\hline DINA657 & 14 & HSM & SEM. DOW & 55000 & 60000 \\
\hline DINACO9560 & 15 & HS & SEM. DOW & 55000 & 65000 \\
\hline 8330 & 16 & HS & ZENECA & 55000 & 65000 \\
\hline \multicolumn{6}{|c|}{ Precoce $-\mathrm{D}_{0}=55000$ plantas ha $^{-1}$} \\
\hline AS32 & 1 & HD & AGROESTE & 52000 & 58000 \\
\hline AS523 & 2 & HD & AGROESTE & 52000 & 58000 \\
\hline AS3477 & 3 & $\mathrm{HT}$ & AGROESTE & 55000 & 60000 \\
\hline AGN2511 & 4 & HTM & AGROMEN & 55000 & 60000 \\
\hline AGN3100 & 5 & HD & AGROMEN & 45000 & 60000 \\
\hline AGN3180 & 6 & HT & AGROMEN & 55000 & 60000 \\
\hline CD301 & 7 & HT & COODETEC & 55000 & 55000 \\
\hline CD3121 & 8 & HS & COODETEC & 60000 & 60000 \\
\hline BRS2160 & 9 & HD & CNPMS & 50000 & 55000 \\
\hline AG122 & 10 & HD & MONSANTO & 45000 & 55000 \\
\hline AG303 & 11 & HD & MONSANTO & 45000 & 55000 \\
\hline AG5011 & 12 & $\mathrm{HT}$ & MONSANTO & 45000 & 60000 \\
\hline C435 & 13 & HD & MONSANTO & 45000 & 55000 \\
\hline C701 & 14 & HD & MONSANTO & 50000 & 55000 \\
\hline DKB280 & 15 & HS & MONSANTO & 50000 & 60000 \\
\hline XL214 & 16 & HS & MONSANTO & 50000 & 60000 \\
\hline XL215 & 17 & HS & MONSANTO & 55000 & 65000 \\
\hline XL344 & 18 & HT & MONSANTO & 50000 & 60000 \\
\hline PREMIUM & 19 & HS & NOVARTIS & 55000 & 65000 \\
\hline TORK & 20 & HS & NOVARTIS & 55000 & 65000 \\
\hline $30 \mathrm{~F} 33$ & 21 & HS & PIONEER & 50000 & 60000 \\
\hline 8447 & 22 & HD & StaHELENA & 50000 & 55000 \\
\hline SHS4040 & 23 & HD & StaHELENA & 50000 & 55000 \\
\hline SHS4050 & 24 & HD & StaHELENA & 55000 & 60000 \\
\hline SHS5050 & 25 & $\mathrm{HT}$ & StaHELENA & 55000 & 60000 \\
\hline SHS5060 & 26 & HT & StaHELENA & 55000 & 60000 \\
\hline SHS5070 & 27 & $\mathrm{HT}$ & StaHELENA & 55000 & 60000 \\
\hline DINA766 & 28 & HSM & SEM. DOW & 55000 & 65000 \\
\hline 8410 & 29 & HS & ZENECA & 55000 & 65000 \\
\hline 8420 & 30 & HS & ZENECA & 55000 & 65000 \\
\hline \multicolumn{6}{|c|}{ Normal $-\mathrm{D}_{0}=50000$ plantas ha $^{-1}$} \\
\hline R-CD303 & 1 & HS & COODETEC & 60000 & 60000 \\
\hline R-OC705 & 2 & HD & COODETEC & 50000 & 50000 \\
\hline R-8550 & 3 & $\mathrm{HT}$ & ZENECA & 55000 & 65000 \\
\hline R-85E03 & 4 & $\mathrm{HT}$ & ZENECA & 55000 & 65000 \\
\hline $\mathrm{R}-3021$ & 5 & HT & PIONEER & 55000 & 65000 \\
\hline CDX98T04 & 6 & $\mathrm{HT}$ & COODETEC & 50000 & 50000 \\
\hline MTC828 & 7 & $\mathrm{HT}$ & MONSANTO & 50000 & 60000 \\
\hline P3232(T) & 8 & $\mathrm{HT}$ & PIONEER & 45000 & 50000 \\
\hline AG1051(T) & 9 & HD & MONSANTO & 45000 & 55000 \\
\hline
\end{tabular}

* HS = Híbrido Simples; HSM = Híbrido Simples Modificado; HD = Híbrido Duplo; HT = Híbrido Triplo; HTM = Híbrido Triplo Modificado. 
Tabela 2 - Graus de liberdade (GL) e quadrado médio para número de dias da semeadura até o pendoamento (DP), estatura de inserção de espigas (EE), estatura de plantas (EP), número de espigas (NE), número de plantas (NP) e rendimento de grãos (REND) para as fontes de variação, média e diferença mínima significativa pelo teste de Tukey para o efeito genótipo $(\Delta \mathrm{g})$ e para o efeito densidade $(\Delta \mathrm{d})$, dos experimentos de genótipo recomendados de milho ciclo superprecoce, precoce e normal nas duas tecnologias. Santa Maria - RS, 2000/2001

\begin{tabular}{|c|c|c|c|c|c|c|c|}
\hline \multirow{2}{*}{ Fontes de variação } & \multicolumn{7}{|c|}{ Quadrado médio } \\
\hline & GL & DP (dias) & $\mathrm{EE}(\mathrm{cm})$ & $\mathrm{EP}(\mathrm{cm})$ & NE (mil ha ${ }^{-1}$ ) & $\mathrm{NP}\left(\mathrm{mil} \mathrm{ha}^{-1}\right)$ & $\operatorname{REND}\left(\mathrm{t} \mathrm{ha}^{-1}\right)$ \\
\hline \multicolumn{8}{|c|}{ Superprecoce - tecnologia melhorada } \\
\hline$\overline{\text { Bloco (B) }}$ & 2 & 4,1 & 916 & 1998 & 157,9 & 50,3 & 2,42 \\
\hline Genótipo (G) & 15 & $73,5 *$ & $1131 *$ & $1926^{*}$ & $263,7 *$ & $75,7^{\text {ns }}$ & $13,23 *$ \\
\hline Erro (g) & 30 & 2,69 & 342,8 & 519,6 & 112,43 & 46,91 & 3,501 \\
\hline Densidade (D) & 1 & $0,0^{\mathrm{ns}}$ & $103^{\text {ns }}$ & $61^{\text {ns }}$ & $40,2^{\text {ns }}$ & $209,3 *$ & $3,77^{\text {ns }}$ \\
\hline$G * D$ & 15 & $0,1^{\mathrm{ns}}$ & $29^{\text {ns }}$ & $45^{\mathrm{ns}}$ & $43,5^{\mathrm{ns}}$ & $82,7^{*}$ & $1,81^{\mathrm{ns}}$ \\
\hline Erro (d) & 32 & 0,052 & 34,4 & 39,0 & 45,14 & 42,13 & 1,369 \\
\hline Média & - & 72,9 & 133,2 & 231,6 & 54,04 & 60,68 & 6,95 \\
\hline$\Delta \mathrm{g}$ & - & 3,53 & 39,83 & 49,04 & 22,81 & 14,73 & 4,025 \\
\hline$\Delta \mathrm{d}$ & - & 0,09 & 2,44 & 2,59 & 2,79 & 2,70 & 0,486 \\
\hline \multicolumn{8}{|c|}{ Superprecoce - tecnologia recomendada } \\
\hline$\overline{\text { Bloco (B) }}$ & 2 & 6,5 & 348 & 495 & 188,9 & 39,6 & 2,95 \\
\hline Genótipo (G) & 15 & $72,2 *$ & $894 *$ & $1169 *$ & $120,8^{*}$ & $31,0 *$ & $7,63^{*}$ \\
\hline Erro $(\mathrm{g})$ & 30 & 3,25 & 236,4 & 282,3 & 49,77 & 12,04 & 1,216 \\
\hline Densidade (D) & 1 & $0,0^{\mathrm{ns}}$ & $36^{\text {ns }}$ & $9^{\text {ns }}$ & $2345,8 *$ & $2563,0 *$ & $16,69 *$ \\
\hline$G * D$ & 15 & $0,1^{\mathrm{ns}}$ & $58^{\mathrm{ns}}$ & $78^{\text {ns }}$ & $44,5^{\text {ns }}$ & $31,1^{*}$ & $1,39^{\mathrm{ns}}$ \\
\hline Erro (d) & 32 & 0,042 & 64,1 & 72,0 & 30,99 & 5,84 & 1,139 \\
\hline Média & - & 72,6 & 130,2 & 225,8 & 52,71 & 58,35 & 6,29 \\
\hline$\Delta \mathrm{g}$ & - & 3,87 & 33,08 & 36,15 & 15,18 & 7,46 & 2,372 \\
\hline$\Delta \mathrm{d}$ & - & 0,08 & 3,33 & 3,53 & 2,31 & 1,00 & 0,443 \\
\hline \multicolumn{8}{|c|}{ Precoce - tecnologia melhorada } \\
\hline Bloco (B) & 2 & 3,1 & 103 & 181 & 3,7 & 12,0 & 4,17 \\
\hline Genótipo (G) & 29 & $34,6 *$ & $932 *$ & $1097 *$ & $70,7 *$ & $26,3 *$ & $4,16^{*}$ \\
\hline Erro (g) & 58 & 3,23 & 42,6 & 54,2 & 15,32 & 3,39 & 1,405 \\
\hline Densidade (D) & 1 & $0,0^{\mathrm{ns}}$ & $87,9^{\text {ns }}$ & $52^{\text {ns }}$ & $679,1 *$ & $990,0 *$ & $1,64^{\text {ns }}$ \\
\hline$G * D$ & 29 & $0,0^{\mathrm{ns}}$ & $30^{\mathrm{ns}}$ & $29^{\text {ns }}$ & $26,2 *$ & $17,2 *$ & $0,73^{\text {ns }}$ \\
\hline Erro (d) & 60 & 0,00 & 35,3 & 36,4 & 13,00 & 4,23 & 0,534 \\
\hline Média & - & 72,7 & 153,2 & 250,7 & 52,73 & 56,19 & 7,34 \\
\hline$\Delta \mathrm{g}$ & - & 4,09 & 14,87 & 16,77 & 8,92 & 4,19 & 2,70 \\
\hline$\Delta \mathrm{d}$ & - & 0,00 & 1,77 & 1,80 & 1,07 & 0,61 & 0,22 \\
\hline \multicolumn{8}{|c|}{ Precoce - tecnologia recomendada } \\
\hline$\overline{\text { Bloco (B) }}$ & 2 & 5,3 & 359 & 741 & 43,3 & 11,4 & 21,26 \\
\hline Genótipo (G) & 29 & $32,9 *$ & $869 *$ & $964 *$ & $64,2 *$ & $20,5 *$ & $3,39 *$ \\
\hline Erro (g) & 58 & 2,49 & 117,7 & 126,0 & 19,99 & 7,20 & 0,900 \\
\hline Densidade (D) & 1 & $0,0^{\mathrm{ns}}$ & $144 *$ & $55^{\text {ns }}$ & $176,8 *$ & $305,8 *$ & $0,82^{\text {ns }}$ \\
\hline$G * D$ & 29 & $0,0^{\mathrm{ns}}$ & $36^{\text {ns }}$ & $28^{\mathrm{ns}}$ & $30,5^{\text {ns }}$ & $17,9^{*}$ & $0,70^{\mathrm{ns}}$ \\
\hline Erro (d) & 60 & 0,017 & 30,3 & 44,7 & 26,64 & 4,18 & 0,662 \\
\hline Média & - & 72,8 & 141,7 & 236,6 & 48,47 & 52,81 & 6,07 \\
\hline$\Delta \mathrm{g}$ & - & 3,59 & 24,71 & 25,57 & 10,18 & 6,11 & 2,161 \\
\hline$\Delta \mathrm{d}$ & - & 0,04 & 1,64 & 1,99 & 1,54 & 0,61 & 0,243 \\
\hline \multicolumn{8}{|c|}{ Normal - tecnologia melhorada } \\
\hline$\overline{\text { Bloco (B) }}$ & 2 & 34,7 & 3236 & 4797 & $48,9^{\mathrm{s}}$ & 19,7 & 7,31 \\
\hline Genótipo (G) & 8 & $32,9 *$ & $1646^{*}$ & $1108 *$ & $1349 *$ & $56,4^{*}$ & $3,10^{*}$ \\
\hline Erro (g) & 16 & 6,62 & 66,5 & 72,3 & 41,36 & 4,66 & 0,971 \\
\hline Densidade (D) & 1 & $0,07^{\text {ns }}$ & $3,5^{\text {ns }}$ & $2^{\text {ns }}$ & $731,0^{*}$ & $744,7 *$ & $12,17 *$ \\
\hline$G * D$ & 8 & $0,03^{\mathrm{ns}}$ & $39^{\text {ns }}$ & $100^{\mathrm{ns}}$ & $45,9^{\text {ns }}$ & $39,6^{*}$ & $1,12^{\mathrm{ns}}$ \\
\hline Erro (d) & 18 & 0,037 & 67,7 & 75,6 & 38,99 & 10,64 & 0,744 \\
\hline Média & - & 77,3 & 130,4 & 225,5 & 45,23 & 54,18 & 5,74 \\
\hline$\Delta \mathrm{g}$ & - & 5,28 & 16,74 & 17,46 & 13,21 & 4,43 & 2,023 \\
\hline$\Delta \mathrm{d}$ & - & 0,11 & 4,70 & 4,97 & 3,57 & 1,86 & 0,493 \\
\hline \multicolumn{8}{|c|}{ Normal - tecnologia recomendada } \\
\hline Bloco (B) & 2 & 13,2 & 1258 & 1132 & $16,5^{\mathrm{s}}$ & 36,4 & 0,97 \\
\hline Genótipo (G) & 8 & $22,6 *$ & $1435^{*}$ & $1259 *$ & $74,6 *$ & $16,3^{*}$ & $4,27 *$ \\
\hline Erro (g) & 16 & 2,87 & 67,9 & $94,1^{*}$ & 29,66 & 6,44 & 0,515 \\
\hline Densidade (D) & 1 & $0,02^{\mathrm{ns}}$ & $64^{\text {ns }}$ & $132^{\text {ns }}$ & $121,2 *$ & $77,7^{*}$ & $0,89^{\text {ns }}$ \\
\hline$G * D$ & 8 & $0,02^{\mathrm{ns}}$ & $26^{\mathrm{ns}}$ & $30^{\text {ns }}$ & $18,6^{\text {ns }}$ & $18,5^{*}$ & $0,67^{\text {ns }}$ \\
\hline Erro (d) & 18 & 0,018 & 49,0 & 54,5 & 29,98 & 3,98 & 0,981 \\
\hline Média & - & 77,2 & 131,1 & 225,8 & 44,90 & 51,55 & 5,85 \\
\hline$\Delta \mathrm{g}$ & - & 3,48 & 16,92 & 19,92 & 11,18 & 5,21 & 1,473 \\
\hline$\Delta \mathrm{d}$ & - & 0,07 & 4,00 & 4,22 & 3,13 & 1,14 & 0,566 \\
\hline
\end{tabular}

* = efeito significativo em nível de 5\% de probabilidade de erro pelo teste $\mathrm{F}$; ${ }^{\text {ns }}=$ efeito não significativo pelo teste $\mathrm{F}$

Ciência Rural, v.35, n.1, jan-fev, 2005. 
Tabela 3 - Média do rendimento de grãos de milho $\left(\mathrm{kg} \mathrm{ha}^{-1}\right)$ dos genótipos de ciclo superprecoce, precoce e normal na condição de tє melhorada (TM) e tecnologia recomendada (TR), média geral, quadrado médio do erro para genótipo (QMe) e coeficiente de (CV). Santa Maria - RS, 2000/2001.

\begin{tabular}{|c|c|c|c|c|c|c|c|c|c|c|c|c|c|c|}
\hline \multicolumn{5}{|c|}{ Superprecoce } & \multicolumn{5}{|c|}{ Precoce } & \multicolumn{5}{|c|}{ Normal } \\
\hline Genótipo & TM & & TR & & Genótipo & $\mathrm{TM}$ & & TR & & Genótipo & TM & & TR & \\
\hline FLASH & 8,978 & $\mathrm{~A}^{*}$ & 7,743 & $A^{*}$ & TORK & 9,112 & $A^{*}$ & 7,033 & $\mathrm{~A}^{*}$ & R-CD303 & 7,094 & $A^{*}$ & 6,536 & $\mathrm{~A}^{*}$ \\
\hline AG6016 & 8,489 & A & 6,905 & A & PREMIUM & 8,981 & $\mathrm{~A}$ & 7,405 & A & P3232(T) & 6,435 & A & 6,349 & A \\
\hline AG6018 & 8,387 & A & 7,079 & A & 8420 & 8,351 & $\mathrm{~A}$ & 6,744 & A & R-85E03 & 6,006 & A & 5,461 & B \\
\hline 32R21 & 8,345 & A & 7,327 & A & XL344 & 8,337 & $\mathrm{~A}$ & 6,243 & A & R-8550 & 5,741 & A & 5,854 & B \\
\hline C806 & 8,072 & A & 7,129 & A & AS523 & 8,198 & A & 6,184 & A & R-3021 & 5,634 & A & 5,710 & B \\
\hline DINACO9560 & 7,756 & A & 6,796 & A & XL214 & 8,178 & A & 6,732 & A & AG1051(T) & 5,488 & A & 5,961 & B \\
\hline 8330 & 7,717 & A & 6,696 & A & SHS5070 & 7,779 & $\mathrm{~A}$ & 6,624 & A & R-OC705 & 5,397 & A & 4,853 & $\mathrm{C}$ \\
\hline AGN3050 & 7,476 & A & 6,666 & A & AGN2511 & 7,754 & $\mathrm{~A}$ & 5,890 & B & MTC828 & 5,294 & A & 7,325 & A \\
\hline AGN3511 & 7,306 & A & 6,850 & A & AG122 & 7,733 & $\mathrm{~A}$ & 6,363 & A & CDX98T04 & 4,574 & A & 4,576 & $\mathrm{C}$ \\
\hline C909 & 7,152 & A & 6,798 & A & 30F33 & 7,721 & $\mathrm{~A}$ & 5,591 & B & & & & & \\
\hline C929 & 5,963 & $\mathrm{~B}$ & 5,596 & B & XL215 & 7,691 & $\mathrm{~A}$ & 6,706 & A & & & & & \\
\hline AGN2012 & 5,732 & B & 6,497 & A & SHS5050 & 7,638 & A & 6,266 & A & & & & & \\
\hline AS3601 & 5,686 & B & 4,344 & $\mathrm{C}$ & AS3477 & 7,594 & A & 5,832 & B & & & & & \\
\hline DINA657 & 5,297 & B & 5,218 & B & AG5011 & 7,563 & $\mathrm{~A}$ & 6,749 & A & & & & & \\
\hline CD3211 & 4,604 & B & 3,768 & $\mathrm{C}$ & DINA766 & 7,514 & $\mathrm{~A}$ & 6,113 & A & & & & & \\
\hline \multirow[t]{15}{*}{ AGN3150 } & 4,237 & B & 5,183 & B & DKB280 & 7,368 & $\mathrm{~A}$ & 6,997 & A & & & & & \\
\hline & & & & & SHS4050 & 7,225 & B & 6,349 & A & & & & & \\
\hline & & & & & AG303 & 7,125 & B & 6,523 & A & & & & & \\
\hline & & & & & AS32 & 7,078 & B & 6,373 & A & & & & & \\
\hline & & & & & CD3121 & 7,009 & B & 5,868 & B & & & & & \\
\hline & & & & & SHS5060 & 6,884 & $\mathrm{~B}$ & 6,127 & A & & & & & \\
\hline & & & & & AGN3100 & 6,874 & B & 5,729 & B & & & & & \\
\hline & & & & & CD301 & 6,671 & B & 5,689 & B & & & & & \\
\hline & & & & & C435 & 6,443 & B & 6,375 & A & & & & & \\
\hline & & & & & AGN3180 & 6,439 & $\mathrm{~B}$ & 4,973 & $\mathrm{C}$ & & & & & \\
\hline & & & & & 8410 & 6,380 & B & 4,212 & $\mathrm{C}$ & & & & & \\
\hline & & & & & 8447 & 6,344 & B & 4,646 & $\mathrm{C}$ & & & & & \\
\hline & & & & & BRS2160 & 6,333 & B & 4,574 & $\mathrm{C}$ & & & & & \\
\hline & & & & & C701 & 6,091 & B & 5,586 & B & & & & & \\
\hline & & & & & SHS4040 & 5,739 & B & 5,496 & B & & & & & \\
\hline Média geral & 6,95 & & 6,29 & & & 7,34 & & 6,07 & & & 5,74 & & 5,85 & \\
\hline QMe & 1,369 & & 1,139 & & & 0,534 & & 0,662 & & & 0,744 & & 0,981 & \\
\hline CV(\%) & 16,8 & & 16,9 & & & 9,9 & & 13,4 & & & 15,0 & & 16,9 & \\
\hline
\end{tabular}

* Genótipos com médias não seguidas por mesma letra diferem pelo teste de Scott-Knott em nível de 5\% de probabilidade de erro.

quais citam que genótipos de ciclo mais curto suportam maior número de plantas e geralmente têm estatura menor. Portanto, as empresas mostraram tendência em recomendar corretamente. No entanto, isto não permite inferir que a recomendação esteja totalmente correta, pois é necessário verificar a magnitude (número de plantas) dessas recomendações.

Caso a interação genótipo densidades fosse significativa, se deveria, sem a menor dúvida, executar os ensaios de competição de genótipos na condição em que cada genótipo tivesse a própria densidade (definida pela empresa produtora da semente). Com o presente estudo, não se pode expor esta recomendação ou porque a definição da densidade de cada genótipo $\left(\mathrm{D}_{\mathrm{i}}\right)$ não foi a mais indicada, ou porque a densidade padrão $\left(\mathrm{D}_{0}\right)$ de cada ciclo foi muito próxima da melhor para todos os genótipos do respectivo ciclo. Contudo, não se deve ignorar a possibilidade de melhorar a qualidade técnica da comparação de genótipos com densidades específicas, pois um melhor agrupamento pode resultar em melhor precisão e, conseqüentemente, na significância da interação genótipo vs densidade para rendimento de grãos.

Ciência Rural, v.35, n.1, jan-fev, 2005. 
Tabela 4 - Média do número de dias da semeadura até o pendoamento (DP), estatura de inserção de espiga (EE), estatura de planta (EP), número de espigas por hectare (NE), número de plantas por hectare (NP) e rendimento de grãos (REND), para densidade $\mathrm{D}_{0}$ (plantas $h^{-1}$ recomendada pela metodologia dos ensaios) e densidade $\mathrm{D}_{\mathrm{i}}$ (plantas/ha recomendada pelos pesquisadores das empresas produtoras de sementes) nos seis experimentos. Santa Maria - RS, 2000/2001.

\begin{tabular}{|c|c|c|c|c|c|c|c|c|}
\hline \multicolumn{2}{|c|}{ Experimentos } & & \multirow{2}{*}{ DP (dias) } & \multirow{2}{*}{$\mathrm{EE}(\mathrm{cm})$} & \multirow{2}{*}{$\mathrm{EP}(\mathrm{cm})$} & \multirow{2}{*}{$\mathrm{NE}$} & \multirow{2}{*}{ NP } & \multirow{2}{*}{$\operatorname{REND}\left(\mathrm{kg} \mathrm{ha}^{-1}\right)$} \\
\hline Tecnologia & Ciclo & & & & & & & \\
\hline Melhorada & SuperPrecoce & $\mathrm{D}_{0}$ & 73,17 & 135,64 & 235,09 & 54253 & 62507 & 6993 \\
\hline Melhorada & SuperPrecoce & $\mathrm{D}_{\mathrm{i}}$ & $73,14^{\mathrm{ns}}$ & $133,23^{\mathrm{ns}}$ & $233,22^{\mathrm{ns}}$ & $52183^{\mathrm{ns}}$ & $57795^{*}$ & $6615^{\mathrm{ns}}$ \\
\hline Melhorada & Precoce & $\mathrm{D}_{0}$ & 72,26 & 149,85 & 244,88 & 51574 & 54074 & 7488 \\
\hline Melhorada & Precoce & $\mathrm{D}_{\mathrm{i}}$ & $72,26^{\mathrm{ns}}$ & $150,69^{\mathrm{ns}}$ & $245,79^{\mathrm{ns}}$ & $57103^{*}$ & $60285^{*}$ & $7754^{\mathrm{ns}}$ \\
\hline Melhorada & Normal & $\mathrm{D}_{0}$ & 77,61 & 127,42 & 222,83 & 42188 & 50694 & 5357 \\
\hline Melhorada & Normal & $\mathrm{D}_{\mathrm{i}}$ & $77,50^{\mathrm{ns}}$ & $127,21^{\mathrm{ns}}$ & $222,50^{\mathrm{ns}}$ & $50795 *$ & $61141^{*}$ & $6396 *$ \\
\hline Recomendada & SuperPrecoce & $\mathrm{D}_{0}$ & 72,58 & 130,79 & 225,53 & 57649 & 63517 & 6704 \\
\hline Recomendada & SuperPrecoce & $\mathrm{D}_{\mathrm{i}}$ & $72,58^{\mathrm{ns}}$ & $129,55^{\mathrm{ns}}$ & $226,13^{\text {ns }}$ & $47762 *$ & 53183* & $5870^{*}$ \\
\hline Recomendada & Precoce & $\mathrm{D}_{0}$ & 73,29 & 143,28 & 241,43 & 49143 & 54300 & 6012 \\
\hline Recomendada & Precoce & $\mathrm{D}_{\mathrm{i}}$ & $73,31^{\mathrm{ns}}$ & $142,29 *$ & $241,23^{\mathrm{ns}}$ & $45872 *$ & $49730 *$ & $5991^{\mathrm{ns}}$ \\
\hline Recomendada & Normal & $\mathrm{D}_{0}$ & 76,50 & 132,18 & 225,07 & 43229 & 50174 & 5802 \\
\hline Recomendada & Normal & $\mathrm{D}_{\mathrm{i}}$ & $76,44^{\mathrm{ns}}$ & $133,83^{\mathrm{ns}}$ & $227,58^{\mathrm{ns}}$ & $47029 *$ & $53426 *$ & $6154^{\text {ns }}$ \\
\hline
\end{tabular}

* $\mathrm{D}_{\mathrm{i}}$ difere de $\mathrm{D}_{0}$ em nível de $5 \%$ de probabilidade de erro pelo teste $\mathrm{F}$; ${ }^{\mathrm{ns}}$ não significativo

Os resultados também revelam a necessidade de estudos mais avançados em termos de densidade ideal para cada genótipo, o que está sendo conseguido pelas empresas responsáveis, em parte, pois, em 49\% (38/78), a densidade D proporcionou menores rendimentos que a densidadè D . Estes resultados concordam com ALMEIDA \& SÅNGOI (1996), MEROTTO JUNIOR et al. (1997), MEROTTO JUNIOR et al. (1999) e ALMEIDA et al. (2000), que obtiveram resposta positiva em termos de rendimento de grãos quando trabalharam com densidades diferentes das recomendadas no estado de Santa Catarina.

Quanto ao aspecto da técnica experimental, não se trata apenas de observar que o rendimento em $\Delta_{\mathrm{i}}$ é melhor e/ou pior do que o rendimento em $\Delta_{0}{ }^{\circ}$ O que deve ser considerado é a variação do rendimento em função da densidade, visto que, com a ausência de uso da densidade correta para um dado genótipo, o resultado do rendimento não se torna justo ou exato, num processo em que se quer comparar os genótipos para fins de indicação aos produtores. Deve-se considerar, ainda, que o custo de variação na quantidade de sementes por unidade de área é muito inferior ao resultado econômico apurado no rendimento de grãos.

Durante a realização dos experimentos no ano agrícola 2000/2001, praticamente não houve deficiência hídrica, mesmo nos experimentos com tecnologia recomendada, nos quais não se aplicou irrigação suplementar. Portanto, essas condições determinaram maior rendimento para as densidades mais elevadas (correlação linear entre número de plantas e rendimento de grãos positiva e significativa, a 5\%). Assim, os resultados obtidos refletem essa realidade, pois, quanto maior o número de plantas, maior o número de espigas e o rendimento, tanto em tecnologia recomendada como em melhorada (Tabela 4).

Diante dos resultados, é possível inferir que a densidade $\mathrm{D}_{0}$ não permite uma avaliação correta dos genótipos de milho nos ensaios de competição, ou seja, impede que alguns genótipos expressem seu potencial em função de estarem sendo avaliados com densidade maior ou menor do que a recomendada pela empresa que o desenvolveu e produziu. Como conseqüência, pode-se estar deixando de sugerir aos produtores um determinado genótipo, que teria um potencial maior do que aqueles indicados. Portanto, o fato de se utilizarem densidades iguais para todos os genótipos dentro do mesmo ciclo não está correto, existindo a necessidade de se avaliarem os genótipos com a densidade capaz de proporcionar o maior rendimento de grãos, cabendo às empresas e às instituições de pesquisa executarem trabalhos de determinação da densidade de plantas em condições diversas, não só tecnológicas, mas também em diferentes regiões e épocas de semeadura e estas densidades devem então ser recomendadas para a avaliação dos genótipos nos ensaios de competição.

\section{CONCLUSÕES}

Os genótipos de milho encontram-se mal agrupados quanto ao ciclo e, por conseqüência, quanto à estatura 
de plantas, aumentando a heterogeneidade nos experimentos e prejudicando a comparação das médias. Genótipos de milho com ciclo e/ou estatura de plantas diferentes não devem ser comparados na mesma densidade e experimento.

\section{REFERÊNCIAS BIBLIOGRÁFICAS}

ALMEIDA, M.L. de; SANGOI, L. Aumento da densidade de plantas de milho para regiões de curta estação estival de crescimento. Pesquisa Agropecuária Gaúcha, Porto Alegre, v.2, n.2, p.179-183, 1996.

ALMEIDA, M.L. et al. Incremento na densidade de plantas: uma alternativa para aumentar o rendimento de grãos de milho em regiões de curta estação estival de crescimento. Ciência Rural, Santa Maria, v.30, n.1, p.23-29, 2000.

EMBRAPA Centro de Pesquisa Agropecuária do Oeste. Milho: informações técnicas. Dourados, 1997. 222p. (Circular Técnica, n.5).

FLESCH, R.D.; VIEIRA, L.C. Espaçamento e população de plantas na cultura do milho. Revista Agropecuária Catarinense, Florianópolis, v.12, n.2, p.28-31, 1999

MARQUES, D.G. As pressuposições e a precisão dos ensaios de competição de cultivares de milho no Estado do Rio Grande do Sul. 1999. $42 \mathrm{f}$. Dissertação (Mestrado em Agronomia) - Universidade Federal de Santa Maria.
MEROTTO JUNIOR, A. et al. Aumento no rendimento de grãos de milho através do aumento da população de plantas. Ciência Rural, Santa Maria, v.27, n.4, p.549-554, 1997.

MEROTTO JUNIOR, A. et al. A desuniformidade de emergência reduz o rendimento de grãos de milho. Ciência Rural, Santa Maria, v.29, n.4, p.595-601, 1999.

PEIXOTO, C. de M. et al. Produtividade de híbridos de milho em função da densidade de plantas, em dois níveis de manejo da água e da adubação. Pesquisa Agropecuária Gaúcha, Porto Alegre, v.3, n.1, p.63-71, 1997

PEREIRA, L.R. et al. Cultivares. In: Indicações técnicas para a cultura de milho no Estado do Estado do Rio Grande do Sul. Porto Alegre : FEPAGRO; EMBRAPA TRIGO; EMATER/RS; FECOAGRO/RS, 2001. Cap.8, p.74-84.

RAMALHO, M.A.P. et al. Experimentação em genética e melhoramento de plantas. Lavras : UFLA, 2000. 326p.

RIBEIRO JÚNIOR, J.I. Análise estatística no SAEG. Viçosa: UFV, 2001. 301p.

SANGOI, L. et al. Desempenho de híbridos de milho com ciclos contrastantes em função da desfolha e da população de plantas. Scientia Agrícola, Piracicaba, v.58, n.2, p.271276, 2001.

SILVA, P.R.F. da et al. Resposta de híbridos de milho irrigado à densidade de plantas em três épocas de semeadura. Pesquisa Agropecuária Brasileira, Brasília, v.34, n.4, p.585-592, 1999.

STORCK, L. et al. Experimentação vegetal. Santa Maria: UFSM, 2000. 198p. 